\title{
Über den Lüneburgit.
}

\author{
Von \\ Wilheim Biltz und E. Marcus.
}

Mit 2 Figuren im Text.

Eine uns vorliegende reine Probe des seltenen Salzlagerstättenminerals Lüneburgit, die wir dem Entgegenkommen Herrn Professor MÜGGES in Göttingen verdanken, gab Veranlassung zu einer Wiederholung der im Jahre 1870 von NosLLNER ${ }^{1}$ angegebenen Analyse. Nach Noeldner ist das Mineral ein wasserhaltiges sekundäres Phosphat und Borat des Magnesiums, das geringe Mengen ron Fluor enthält. Seine Zahlen entsprechen, wenn auch keineswegs genau, der Bruttoformel $3 \mathrm{MgO} \cdot \mathrm{P}_{2} \mathrm{O}_{5} \cdot \mathrm{B}_{2} \mathrm{O}_{3} \cdot 8 \mathrm{H}_{2} \mathrm{O}$. Da weder in seiner $\mathrm{Ab}$ handlung Näheres über die Ausführung der Analyse mitgeteilt ist ein Bestandteil scheint aus der Differenz berechnet zu sein - noch seither eine Nachprüfung der Zusammensetzung vorgenommen worden ist, so schienen auch in Anbetracht der analytisch nicht ganz landläufigen Aufgabe Bedenken gegenüber der Zuverlässigkeit der Zahlen möglich. Wie vorweg betont sei, haben sich diese indessen als ganz unbegründet erwiesen.

Zur Wasserbestimmung wurde die Substanz mit Soda geglüht und das entwichene Wasser direkt gewogen. In der Hauptanalyse wurde zunächst die Borsäure als Methylester verflüchtigt und als $\mathrm{B}_{2} \mathrm{O}_{3}$ gewogen. Im Rückstand bestimmte man Phosphorsäure und Magnesium. Eine gewisse Kontrolle der Phosphorsäure- und Borsäuredaten lieferte die Titration, über die an zweiter Stelle berichtet wird.

Bei der Borsäurebestimmung schlossen wir uns im wesentlichen dem von H. BILTZ ${ }^{2}$ in Gemeinschaft mit E. Topp benutzten und be-

${ }^{1}$ Münch. Akad. Ber. 1570, 291. Vgl. Vogter, Tageblatt der 45. Verslg. Deutscher Naturf. u. Ärzte 1S72, 60. Als Mutterlaugenbildung bei Lüneburg fand sich Lüneburgit, der nach Noeldoner eine eigentümlich konstituierte Verbindung von ca. $25 \% \mathrm{MgO}, 30 \% \mathrm{P}_{2} \mathrm{O}_{3}, 15 \% \mathrm{~B}_{2} \mathrm{O}_{3}$ und $30 \% \mathrm{H}_{2} \mathrm{O}$ ist. „Es sind weiße Knollen; die in größerer Tiefe sind kristallisiert, blättrig oder fasrig, äbulich dem Fasergips. Sehr häufig kommen Knollen von charakteristischer Form vor, die jerlenfalls den Rest einer Śpongie darstellen."

2 Ber. 41 (1908), 2634; 43 (1910), 297. 
fürworteten Verfahren an. In einem Destillierkolben mit aufgesetztem Tropftrichter und angeschmolzenem Kühler wurde die Probe in verdünnter Salpetersäure gelöst; ein Rückstand blieb dabei nicht. Die als Vorlage dienende gekühlte Saugflasche und die weiterhin vorgelegte VoLHARDsche KugelrohrHasche waren mit konzentrierter Ammoniakflüssigkeit beschickt. Zuerst wurde die Salpetersäure zum gröBten Teile übergetrieben und dann wurde $8 \mathrm{mal}$ mit je $10 \mathrm{ccm}$ Methylalkohol destilliert. Die Flammenprobe mit dem zuletzt übergehenden Tropfen verlief negativ. Zur Auswage der Borsäure wurde bei Verwendung von $0.2 \mathrm{~g}$ Mineral ein Platintiegel mit $2.5 \mathrm{~g} \mathrm{CaO}$ vorbereitet, indem man die nötige Menge Calciumkarbonat (Kahlbaum) im Sauerstoffstrome durch zweimal je einstündiges Glühen bei ca. $1000^{\circ}$ (H eraeus - Tiegelofen) zu konstantem Gewichte brachte. Dann ließ man in den auf einem langsam siedenden Wasserbade stehenden Platintiegel das alkalische Destillat aus einem Tropftrichter allmählich in dem MaBe eintropfen, wie die Flüssigkeit verdampfte. Eine Ansammlung von Flüssigkeit im Tiegel ist durchaus zu vermeiden. Der Tiegelinhalt wurde bei einer von $100^{\circ}$ auf $160^{\circ}$ steigenden Temperatur getrocknet und gelinde mit freier Flamme geglüht. Dabei trat stets eine schwache Grünfärbung der Flamme am Tiegelrande auf; ein geringer Borverlust ist hier also nicht zu vermeiden. Schließlich glühte man, wie vorher, im Tiegelofen zu konstantem Gewicht. Ein Zusatz von Schwefelsäure bei der Veresterung und Destillation der Borsäure erwies sich als nicht ratsam, da hierbei schweflige Säure in das Destillat übergebt, die sich als Calciumsulfat in der Auswage wiederfindet.

Zur Scheidung des Magnesiums von der Phosphorsäure wurde der Kolbeninhalt mit Ferrinitrat versetzt, das aus zehnmal soviel Eisen bereitet war, wie sich für Ferriphosphatbildung als nötig berechnet. Nach zweimaliger Anwendung der Acetatmethode war die Trennung vollständig. Eine Fällung mit Ammoniak ist hier auch an zweiter Stelle wegen der fällenden Wirkung der Phosphorsäure auf das Magnesium zu vermeiden. In dem Eisenniederschlag bestimmte man die Phosphorsäure nach Abscheidung mit Molybdat, wie üblich, als Magnesiumpyrophosphat. Im Filtrate wurde das Magnesium in eben dieser Form bestimmt und das ausgewogene Pyrophosphat nach Huluebrand auf Calcium geprüft.

0.6285 g Substanz ergaben $0.2021 \mathrm{~g} \mathrm{H}_{2} \mathrm{O}$.

$0.2021 \mathrm{~g}$ Substanz ergaben $0.0260 \mathrm{~g} \mathrm{~B}_{2} \mathrm{O}_{3} ; 0.0938 \mathrm{~g} \mathrm{Mg}_{2} \mathrm{P}_{2} \mathrm{O}_{7}$ (für $\mathrm{P}_{2} \mathrm{O}_{5}$ ); $0.1402 \mathrm{~g} \mathrm{Mg}_{2} \mathrm{P}_{2} \mathrm{O}_{7}$ (für $\mathrm{Mg}$ ) und $0.0003 \mathrm{~g} \mathrm{CaO}$. 


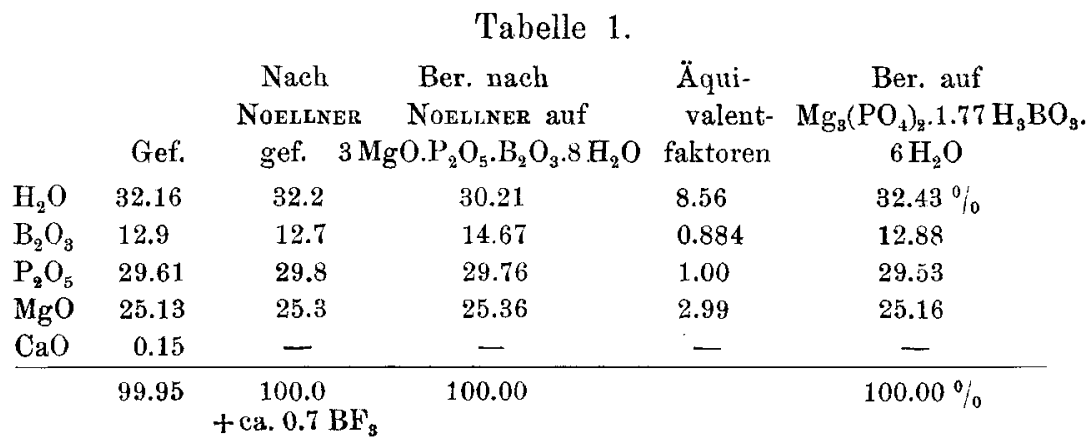

Wie die Addition unserer Werte zeigt, können Fluor und etwaige sonstige Bestandteile nur in ganz untergeordneter Menge vorliegen. Zur Prüfung auf Fluor eignet sich die jüngst veröffentlichte, ${ }^{1}$ uns sonst als beste bekannte Methode von Browning wegen der Anwesenheit der Borsäure hier nicht. Wir griffen daher auf die alte und wohl vielfach in Vergessenheit geratene Probe ${ }^{2}$ der Lötrohrprobierkunst zurück, die Fluor an der Gelbfärbung ron Fernambukpapier erkennen läßt, wenn man die Probe mit Natriummetaphosphat in geeigneter Weise erhitzt. Sehr geringe Mengen ron Fluor lieBen sich danach im Lüneburgit nachweisen. Eisen enthält der Lüneburgit auch nicht spurenweise.

In sehr einfacher, wenn auch nicht so zuverlässiger Weise, als gewichtsanalytisch, läßt sich die Phosphorsäure- und Borsäurebestimmung im Lüneburgit titrimetrisch vornehmen. Näheres über die Grundlagen dieses Verfahrens wird in der nachstehenden Mitteilung berichtet. $0.2307 \mathrm{~g}$ Mineral wurden in Salzsäure gelöst und unter Verwendung von Methylorange als Indikator mit Natronlauge neutralisiert. Mit Phenolphtalein titriert sich nunmehr die vorliegende Phosphorsäure einbasisch, während die Borsäure nicht oder nur zu geringem Teile neutralisiert wird. Fügt man jetzt Mannit hinzu und titriert bis zur bleibenden Rotfärbung, so entspricht die bei dieser letzten Titration verbrauchte Natronlauge annähernd der vorhandenen Borsäure. $0.2307 \mathrm{~g}$ Substanz verbrauchten so für Phosphorsäure $9.50 \mathrm{ccm}^{1} / 10^{-n o r m}$. NaOH; für Borsäure $8.60 \mathrm{ccm}^{1} / 10^{-n o r m}$. $\mathrm{NaOH}$. Gef. $\mathrm{B}_{2} \mathrm{O}_{3} 13.05 ; \mathrm{P}_{2} \mathrm{O}_{5} 29.2 \%$.

Schlieblich konnte man die beiden letzten Titrationen vereinigen, indem man die gegen Methylorange neutrale Lösung sofort

1 Z. anorg. Chem. 74 (1912), 86.

${ }^{2}$ Pratriner-Kolbeck, Probierkunst mit dem Lötrohre 1907, 7. Aufl, S. 375. 
mit Mannit und Phenolphtalein titrierte und unter Hinzuziehung des gewichtsanalytisch bestimmten Gehaltes an $\mathrm{B}_{2} \mathrm{O}_{3}$ aus der Differenz den $\mathrm{P}_{2} \mathrm{O}_{5}$-Gehalt ermittelte oder umgekehrt. $0.2325 \mathrm{~g}$ Substanz verbrauchten so $18.20 \mathrm{~cm} 1 / 10$-norm. NaOH. $12.9 \%$ laut Gewichtsanalyse vorhandener Borsäure entsprechen $8.57 \mathrm{ccm} 1 / 10^{-}$-norm. $\mathrm{NaOH}$. Für die Phosphorsäure blieben also $9.63 \mathrm{ccm} 1 / 10^{-n o r m}$. $\mathrm{NaOH}=29.4 \% \mathrm{P}_{2} \mathrm{O}_{5}$.

In der vorliegenden Bestätigung der NokLLNer schen Analyse ist ein Fortschritt insofern zu erblicken, als die von ihm gegebene Formel nunmehr unhaltbar wird. Denn die Unterschiede (vgl. Tabelle 1), die hiernach die Rechnung gegenüber der Beobachtung zeigt, sind viel größer, als die bestätigte Analyse zuläßt oder als Zufälligkeiten in der Zusammensetzung der beiderseitig vorliegenden Mineralproben erklären. Das Mineral erscheint vielmehr von sehr konstanter Zusammensetzung. Die Berechnung der Äquivalentfaktoren auf Grund unserer Analysenzahlen liefert kein auf den ersten Blick übersichtliches Bild; man würde, wenn man nicht auf die Forderung ganzzahliger Äquivalentmultipla zunächst verzichtete, zu außerordentlich komplizierten, vom chemischen Standpunkte sehr unwahrscheinlichen Formeln gelangen. Dagegen fällt auf, daß das Äquivalentverhältnis von $\mathrm{P}_{2} \mathrm{O}_{5}: \mathrm{MgO}$ recht genau gleich $1: 3$ ist; und daß ferner der Faktor für Wasser zu dem für $\mathrm{P}_{2} \mathrm{O}_{5}$ sich nahe, wie $6: 1$ verbält, wenn von dem Wasserfaktor soviel subtrahiert wird, wie zur vollständigen Hydratisierung des gefundenen $\mathrm{B}_{2} \mathrm{O}_{3}$ nötig ist. Hiernach läge also erstens $1 \mathrm{Mol}$ tertiären Magnesiumphosphats vor, zweitens 6 Mole Wasser und drittens Orthoborsäure. Die Schwierigkeit besteht nur darin, daB der zuletzt genannte in kein einfaches ganzzahliges Verhältnis zu den zuerstgenannten Bestandteilen zu bringen ist. Die Formel würde lauten $\mathrm{Mg}_{3}\left(\mathrm{PO}_{4}\right)_{2}$. $1.77 \mathrm{H}_{3} \mathrm{BO}_{3} .6 \mathrm{H}_{2} \mathrm{O}$ und hiermit den gefundenen Werten befriedigend entsprechen.

Daß 6 Mole Wasser eine Sonderstellung in diesem Mineral einnehmen, zeigt unmittelbar die systematische Entwässerung; und gleichzeitig wird dadurch aufs neue wahrscheinlich gemacht, daß das übrige Wasser als Borsäure gebunden ist.

$0.3798 \mathrm{~g}$ Substanz wurden in Zimmerluft nacheinander auf jede der in Tabelle 2 angegebenen Temperaturen im Trockenschrank oder im Heraeustiegelofen solange erhitzt, bis zwei aufeinanderfolgende Wägungen um nicht mehr als $1 \mathrm{mg}$ differierten.

Selbstverständlich ist dies nur ein Näherungsverfahren, das durch 
Tabelle 2.

\begin{tabular}{c|c|c|c}
\hline \hline$t^{0}$ & $\%$ Abnahme & $t^{0}$ & $\%$ Abnahme \\
\hline \hline 100 & 0.08 & 327 & 28.2 \\
110 & 0.10 & 350 & 28.2 \\
120 & 0.10 & 375 & 28.9 \\
140 & 0.16 & 400 & 29.0 \\
160 & 0.18 & 425 & 29.7 \\
180 & 0.26 & 450 & 30.2 \\
200 & 0.32 & 505 & 30.3 \\
225 & 21.7 & 600 & 32.6 \\
250 & 22.8 & 700 & 32.9 \\
275 & 26.7 & 800 & 33.0 \\
300 & 27.4 & &
\end{tabular}

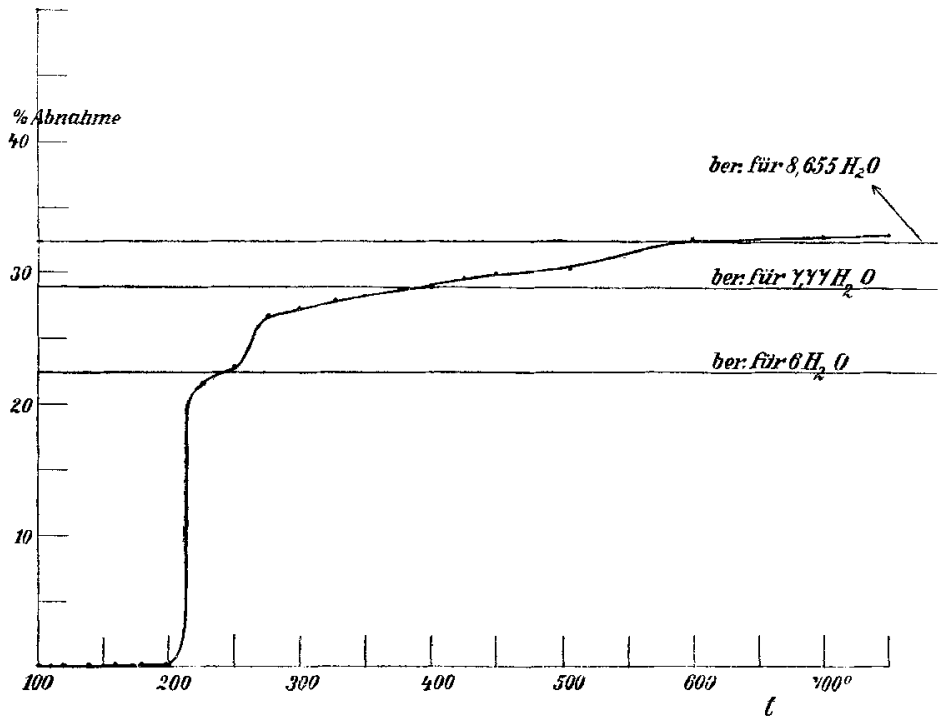

Fig. 1.

Versuche in einer Atmosphäre konstanter Wassertension vervollkommnet werden kann; aber es genügt zu zeigen, daß nach Verlust von $6 \mathrm{Mol}$ Wasser $(22.5 \%)$ eine Verlangsamung der weiteren Wasserabgabe stattfindet; ein Gebiet weiterer Verlangsamung findet sich bei im Mittel $29.1 \%$ Wasser, dem Übergange der vorhandenen Orthoborsäure in Metaborsäure entsprechend und Gewichtskonstanz wird bei vollständiger Entwässerung der Borsäure $(32.4 \%$ ) erreicht.

Für die Konstitutionsaufklärung des Lüneburgits bleibt nur noch übrig, zu entscheiden, welche Funktion die Borsäure neben 
dem tertiären Magnesiumphosphat hat. Entweder kann sie mechanisch beigemengt sein oder es können Mischkristalle beider Stoffe vorliegen oder es besteht zwischen beiden eine chemische Bindung.

Der mikroskopische Befund und die Konstanz in der Zusammensetzung rerschiedener Lüneburgitproben machen das erste unwahr. scheinlich. Besondere Versuche, bei denen Lüneburgitpulver $1 / 2$ Stunde unter Rückfluß mit Alkohol oder Wasser gekocht wurden, zeigten, daß die Borsäure nicht als freier Gemengteil vorliegen kann; denn es gingen an das Extraktionsmittel nie mehr als Spuren titrierbarer Borsäure über.

Tertiäres Magnesiumphosphat findet sich in der Natur als Wagnerit, ein im übrigen noch fluorhaltiges Mineral. Man könnte meinen, es lägen im Lüneburgit Mischkristalle von tertiären Magnesiumphosphat und Borsäure vor. Über die mikroskopische Prüfung, teilte uns Herr MüGGE freundlichst das Folgende mit:

„Die nur bis ca. $0.1 \mathrm{~mm}$ großen Individuen sind anscheinend monoklin; Tafeln mit roh hexagonalemUmriß spalten parallel

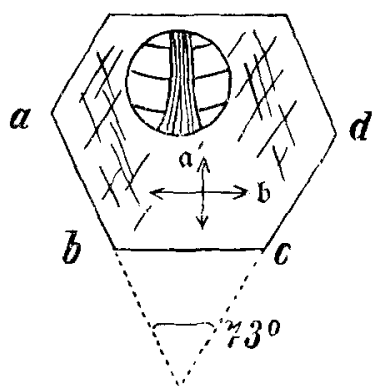

Fig. 2. den Kanten $a b$ und $c d$ (Winkel ca. $73^{\circ}$ ); feine Streifung auf der Tafelfläche $\| a b$ und $c d$; sehr milde, feinschuppige Klümpchen. Ebene der optischen Achsen $\perp b c$, Achsenwinkel anscheinend nur klein; Doppelbrechung negativ; Bisectrix stark geneigt zur Tafelfläche; in der Tafelfläche $\| l c$ die kleinere optische Elastizitätsachse (b); die (dünneren) einheitlichen Blättchen zeigen Interferenzfarben bis Orange I. Ordnung; dickere sind fast nie homogen, sondern hypoparallel verwachsen. Brechungsindex ungefähr 1.53. Nach stärkerem Erhitzen trüb bräunlichgelb (nur Trübungsfarbe?!), zerfällt in mehrere Systeme feiner Streifen (Aggregatpolarisation)."

Borsäure kristallisiert triklin, aber der Habitus der Kristalle ähnelt dem hier beobachteten, wenngleich die Winkelverschiedenheiten nicht unbeträchtlich sind. Es bleibt also fraglich, ob die Bildung von Mischlrristallen vom kristallographischen Standpunkte hier wahrscheinlich wird.

Es besteht aber ferner die Möglichkeit, daß die Borsäure dem Magnesiumphosphat chemisch zugeordnet ist, daß der Lüneburgit also das Hexahydrat des tertiären Magnesiumsalzes einer komplexen Borophosphorsäure: $\mathrm{Mg}_{3}\left[\left(\mathrm{PO}_{4}\right)_{2}\left(\mathrm{H}_{3} \mathrm{BO}_{3}\right)_{1.77}\right] \cdot 6 \mathrm{H}_{2} \mathrm{O}$ ist. 
Salze von diesem l'ypus kennt man bisher nicht. Wohl aber wird ein Salz: $\mathrm{Na}_{4} \mathrm{P}_{2} \mathrm{O}_{7}, \mathrm{~B}_{2} \mathrm{O}_{3}{ }^{1}$ genannt und eine Verbindung $\mathrm{B}_{2} \mathrm{O}_{3}$. $\mathrm{P}_{2} \mathrm{O}_{5}{ }^{2}$ beschrieben und die Hähigkeit der Borsäure sich mit anderen Säuren zu Heteropolysüuren zu vereinigen, ist bekannt. Gerade auf diesem Gebiet ist man an Zusammensetzungen mit hohen und auffallenden Äquivalentfaktoren gewöhnt und man würde daher mit der Zureclnung des Lüneburgits zu dieser Verbindungsklasse seinen Charakter treffen, wenn synthetische Versuche die vorläufig noch naheliegende Möglichkeit der Mischkristallbildung zwischen den beiden Mineralkonstituenten ausschlössen.

Der Verband zur wissenschaftlichen Erforschung der deutschen Kalisalzlagerstätten unterstützte uns in dankenswerter Weise in der Fortführung unserer Arbeiten.

${ }^{1}$ Apecos Handbuch III $_{1}$, S. 35.

2 G. Meyer, Ber. 22 (1889), 2919; vgl. F. Mylus und A. Mrusser, Ber. 37 (1904), 397 .

Clausthal i. H., Irgl. Betriebslaboratorizm.

Bei der Redaktion eingegangen am 25. Mai 1912. 